This is the peer reviewed version of the following article:

Lanzani, G., Susi, T., Ayala, P., Jiang, T., Nasibulin, A. G., Bligaard, T., Pichler, T., Laasonen, K. and Kauppinen, E. I. (2010),

Mechanism study of floating catalyst CVD synthesis of SWCNTs.

phys. stat. sol. (b), 247: 2708-2712,

which has been published in final form at https://doi.org/10.1002/pssb.201000226.

This article may be used for non-commercial purposes in accordance with Wiley Terms and Conditions for Use of Self-Archived Versions. 


\title{
Mechanism study of floating catalyst CVD synthesis of SWCNTs
}

\author{
Giorgio Lanzani $i^{*}, 1,2$, Toma Susi ${ }^{3}$, Paola Ayala ${ }^{4}$, Tao Jiang ${ }^{5}$, Albert G. Nasibulin ${ }^{3}$, Thomas Bligaard ${ }^{5}$ \\ Thomas Pichler ${ }^{4}$, Kari Laasonen ${ }^{* *, 2}$, and Esko I. Kauppinen ${ }^{* * *, 3}$ \\ ${ }^{1}$ Thule Institute, University of Oulu, P.O. Box 7300, 90014, University of Oulu, Finland \\ ${ }^{2}$ Department of Chemistry, University of Oulu, P.O.Box 3000, 90014, University of Oulu, Finland \\ ${ }^{3}$ Department of Applied Physics, Aalto University School of Science and Technology, P.O.Box 15100, 00076 AALTO, Finland \\ ${ }^{4}$ Fakultat für Physik, Universität Wien, Strudlhofgasse 4, 1090 Wien, Austria \\ ${ }^{5}$ Center for Atomic scale Materials Design, Technical University of Denmark, Building 311, 2800 Kongens Lyngby, Denmark
}

Received ZZZ, revised ZZZ, accepted ZZZ

Published online ZZZ (Dates will be provided by the publisher.)

Keywords CVD, nanoparticle, DFT, mechanism, synthesis

* Corresponding author: e-mail giorgio.lanzani@oulu.fi, Phone: +358 40876 4593, Fax: +358 85531603

** e-mail kari.laasonen@oulu.fi, Phone: +358 8553 1640, Fax: +358 85531603

*** e-mail esko.kauppinen@tkk.fi, Phone: +358 94702 8250, Fax: +358 924513517

Catalysis over metal nanoparticles is essential for carbon nanotube growth. Thus it is very important to understand the carbon chemistry on nanometer size metal particles. First-principles electronic-structure calculations have been used to investigate $\mathrm{CO}$ disproportionation on an isolated $\mathrm{Fe}_{55}$ cluster. After $\mathrm{CO}$ dissociation, $\mathrm{O}$ atoms remain on the surface while $\mathrm{C}$ atoms move into the cluster, presumably as the initial step toward carbide formation. The lowest $\mathrm{CO}$ dissociation barrier found on the cluster $(0.63 \mathrm{eV})$ is lower than on most studied Fe surfaces. The dissociation occurs on a vertex between the facets. A possible path for $\mathrm{CO}_{2}$ formation was also identified with a lowest reaction barrier of $1.04 \mathrm{eV}$.

1 Introduction Carbon nanotubes (CNTs) are of great interest since they exhibit unique and useful chemical and physical properties related to toughness, electrical/thermal conductivity and magnetism [1]. Several techniques have been introduced to synthesize CNTs [2-5], but among the various routes, chemical vapour deposition (CVD) methods have attracted widespread interest since they enable highly controlled and continuous CNT production on designed architectures [5-8]. In these processes, metal nanoparticles are produced in a mixed flow of carbon precursors and other gases (e.g., hydrogen). The growth process is driven by cleaving the carbon atoms from the precursors and these atoms will form carbon structures on the nanoparticles' surface. The metal catalyst nanoparticle is essential for CNT growth. All properties, like diameter and chirality, of the nanotube are mostly determined by the particle. Control of the CNTs' properties is essential for their use in technological applications [9]. Thus it is very important to understand the carbon chemis-

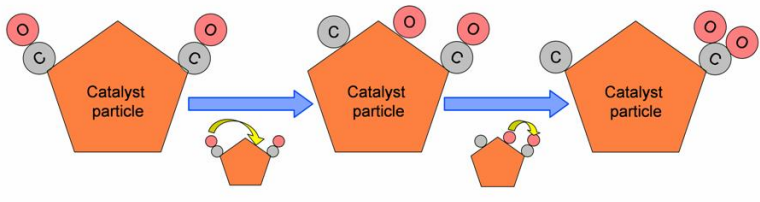

Proposed carbon monoxide disproportionation mechanism ( $\mathrm{Fe}$, brown; $\mathrm{C}$, grey; and $\mathrm{O}$, red).

try on nanometer size metal particles. Carbon monoxide (CO) is one of the most common carbon sources for the production of CNTs [10-12]. This is due to the reasonable temperature range for $\mathrm{CO}$ disproportionation and the good CNT yield. In the ideal case, the disproportionation of $\mathrm{CO}$ is the only reaction needed for the production of CNTs (Figure in the abstract). This is not possible in practice, because the diffusion of carbon through the catalyser surface is generally considered to be the rate-limiting step of the overall process. Since the diffusion of carbon species through the crystal from the gas/metal interface to the metal/carbon interface is the rate limiting step in CNT formation, the rate of carbon atoms produced on the catalyst surface may exceed the rate of subsequent segregation and diffusion of carbon atoms in the particle, which leads to the accumulation of carbon on the catalyst surface.

The literature regarding the synthesis of single and multiwalled CNTs is abundant, but still far from being fully comprehended. It is clear, that in the CVD process, car- 
bon saturates the catalyst whatever its nature may be However, it is argued that the catalyst both nucleates a carbon nanotube and sustains growth. In addition, the catalyst is believed to catalytically decompose the carbon feedstock. In CVD, Fe is a highly effective catalyst. However, it should be borne in mind that hydrogen is commonly present due to the use of decomposing hydrocarbons and often the addition of pure $\mathrm{H}_{2}$ or $\mathrm{NH}_{3}$ is also implemented. There is some controversy with iron over its oxidation state, but this is not the matter of discussion in this contribution.

To understand the CNT formation chemistry on a catalytic particle, several reactions need to be considered. Thus a complete study with all the involved species is probably an unreachable goal with the present computational resources. For these reasons, the present study is addressing the disproportionation of isolated $\mathrm{CO}$ molecules on iron nanoclusters $\left(\mathrm{CO}_{(\mathrm{g})}+\mathrm{CO}_{(\mathrm{g})} \leftarrow \rightarrow \mathrm{CO}_{2(\mathrm{~g})}+\mathrm{C}_{(\mathrm{s})}\right)$ and the mobility of atomic carbon. The detailed chemistry on the surface of CNT catalyst transition-metal nanoparticles is largely unknown, but it is believed that the adsorbed $\mathrm{CO}$ first dissociates, $\mathrm{CO}_{(\text {ads })} \rightarrow \mathrm{C}_{(\text {ads })}+\mathrm{O}_{(\text {ads })}$, and the $\mathrm{O}$ will react with an undissociated $\mathrm{CO}$ to form surface $\mathrm{CO}_{2}$ (ads).

In our previous studies [13], the reactivity of $\mathrm{CO}$ on a nanosized iron cluster has been tested using density functional theory (DFT) calculations with the PW91's generalized gradient approximation (GGA-PW91) for the exchange and correlation functional [14]. The calculations were performed with the Vienna ab initio simulation package (VASP code [15]) with the projector augmented wave (PAW) method [16]. Although the obtained results present good agreement with experimental results, the dimension of the simulated and experimentally used clusters differed significantly. Grid-based PAW (GPAW) [17] is also a density-functional Python code based on the projectoraugmented wave method [16], but it uses real-space uniform grids and multigrid methods or atom-centred basisfunctions. The usage of real space grids allows drastically reducing the computational cost. Based on these considerations, it could be convenient to study the reactivity mechanism described above on a cluster bigger than the $\mathrm{Fe}_{55}$ with the GPAW code. Before studying a realistic-size cluster, it is important to verify the capability of the code to reproduce the earlier results. In order to test the reliability of the GPAW code for the description of the cluster's role in this reaction, we have studied the different steps of the $\mathrm{CO}$ reaction on the $\mathrm{Fe}_{55}$ nanocluster. The computational work is combined with experimental investigations of the same reaction on larger nanoclusters [12]. Surface analysis of the iron oxide/carbide nanoparticles collected after the reaction were performed by X-ray photoelectron spectroscopy (XPS).

2 Methods The method for SWCNT production has been described in detail elsewhere [12]. In the method, premade catalyst particles of controlled size, concentration and polydispersity are introduced into well defined synthe- sis conditions. Briefly, in this method, catalyst particles were generated by vaporization of a resistively heated iron catalyst wire (HWG). Subsequently, the particles were introduced into a heated ceramic tube reactor and mixed with a CO flow to induce CNT formation. This process differs significantly from the one presented in our previous studies in which the nanoparticle formation was based on thermal decomposition of ferrocene [13].

XPS spectra were recorded on the as grown material using a PHI 5600 spectrometer equipped with a monochromatic Al KR source (1486.6 eV).

For electronic structure calculations (GPAW code), we have used DFT with the RPBE's generalized gradient approximation (GGA-RPBE) for the exchange and correlation functional $[18,19]$. Iron has a large magnetic moment, and in the cluster geometry, the spin orientation is subtle. Therefore, we have fully incorporated the effects of spin polarization and collinear magnetic moments in our calculations [13]. The adequacy of the computational parameters has been tested against the calculation of cluster geometry. These settings are also used for the rest of calculations. The reaction barriers are more demanding to study and special transition-state search algorithms, like NEB [20], have been used for their determination.

\section{Results}

3.1 Kinetic investigations The CO disproportionation reaction was investigated in a horizontal quartz tube at a heating rate of $5^{\circ} \mathrm{C} /$. Here, a silica substrate with $2.8 \pm 0.6$ $\mathrm{nm}$ sized iron particles deposited from the HWG reactor was placed inside the tube. The $\mathrm{CO}$ flow rate through the tube was maintained at $8.3 \mathrm{~cm}^{3} / \mathrm{min}$. The concentration of the gaseous product $\left(\mathrm{CO}_{2}\right)$ was monitored on-line by infrared (IR). The region of $\mathrm{CO}_{2}$ concentration increase from about $350{ }^{\circ} \mathrm{C}$ to about $580{ }^{\circ} \mathrm{C}$ is the kinetic region, where the rate of the $\mathrm{CO}$ disproportionation reaction is significant. At temperatures above $600{ }^{\circ} \mathrm{C}$, the reaction is limited thermodynamically. Since the disproportionation rate is proportional to the concentration of $\mathrm{CO}_{2}$, the activation energy of the reaction can be found using an Arrhenius dependence: $\mathrm{X}\left(\mathrm{CO}_{2}\right)=\mathrm{k}_{0} \exp \left(-\mathrm{E}_{\mathrm{a}} / \mathrm{RT}\right)$, where $\mathrm{X}\left(\mathrm{CO}_{2}\right)$ is the carbon dioxide mole fraction. Plotting the kinetic region in the coordinates of $\ln \mathrm{X}\left(\mathrm{CO}_{2}\right)$ versus $1 / \mathrm{T}$, one gets $\mathrm{E}_{\mathrm{a}}=31.6$ $\mathrm{kJ} / \mathrm{mol}=0.33 \mathrm{eV}$ as the barrier for the $\mathrm{CO}$ disproportionation reaction (Figure 1).

3.2 XPS Analysis Figure 2 shows an XPS spectrum of the raw material collected at the reactor outlet. The inset of the figure shows an overall survey, which shows the expected core level signals of $\mathrm{C}$ and $\mathrm{O}$, as well as the signatures for $\mathrm{Fe} 2 \mathrm{p}_{1 / 2}$ and $\mathrm{Fe} 2 \mathrm{p}_{3 / 2}$. The well-defined Fe $2 \mathrm{p}_{3 / 2}$ peak located at $706.8 \mathrm{eV}$ suggests the presence of metallic $\mathrm{Fe}$ or in $\mathrm{Fe}$ in an oxidation state of +3 . 


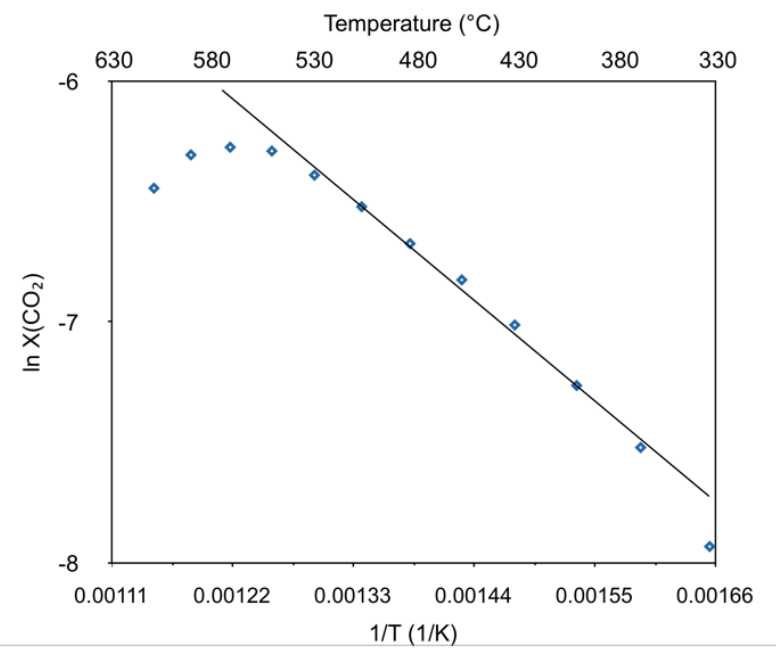

Figure 1 Kinetic region of $\mathrm{CO}$ disproportionation reaction fitted with a line (Arrhenius plot).

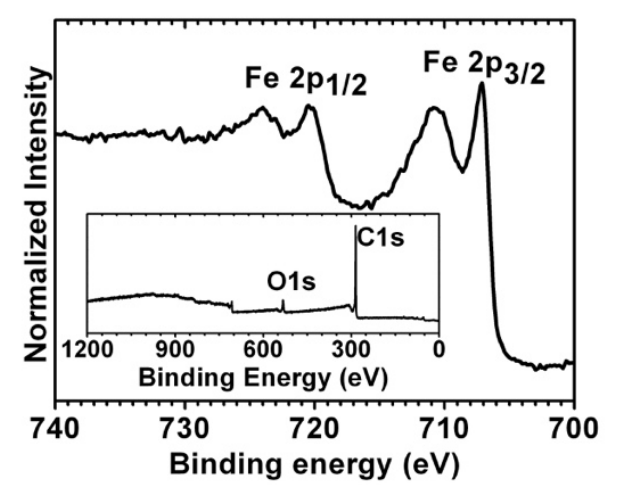

Figure 2 XPS spectrum recorded on a catalyst particle collected in the post-synthesis zone of the experimental apparatus. The $2 p$ levels signals are shown in the main panel. The inset shows a broad survey scan of the probed material.

3.3 Theoretical calculations Based on CO adsorption and $\mathrm{C} / \mathrm{O}$ co-adsorption data, a possible dissociation path for CO was mapped out with the NEB method (Figure 3). This reaction is exothermic by $0.12 \mathrm{eV}$. In the transition state, the $\mathrm{C}-\mathrm{O}$ bond is elongated to $1.30 \AA$, with the $\mathrm{O}$ atom close to a defect of the surface (in this case the edge of the cluster). This type of transition state is common for $\mathrm{CO}$ dissociation on transition metal surfaces [21-24]. The predicted path for $\mathrm{CO}$ dissociation is similar compared to calculations on other surfaces, but the presence of the vertex lowers the barrier. The barrier of $0.63 \mathrm{eV}$ obtained in this calculation is significantly lower than on most reported surfaces (the lowest case is $0.78 \mathrm{eV}$ [23]), suggesting that $\mathrm{Fe}_{55}$ is much more reactive toward $\mathrm{CO}$ dissociation than most stepped Fe surfaces. It seems that the ability of the metal atoms to activate reactants changes substantially as their coordination is reduced both on the atomic steps and the vertices. The obtained results show that the catalytically most active sites are the vertices where the molecules also have a relatively low coordination number.

The mechanism for $\mathrm{CO}$ oxidation involves a reaction of the $\mathrm{CO}$ molecule and oxygen atom adsorbed on the surface. The structures of all transition states and intermediates are depicted in Figure 4 together with the energy profile. The obtained formation energy is $+0.62 \mathrm{eV}$ with a barrier of $+1.04 \mathrm{eV}$. Considering that the particle morphology and reaction conditions are different, the calculated data are in reasonable agreement with the experimental results of Li et al. [28] and earlier results by the authors [13]. The experimentally measured barriers are in good agreement with experimental results for the $\mathrm{Fe}_{2} \mathrm{O}_{3}$ catalysts used in modern industrial applications [25]. The formation of an oxide phase is confirmed by our XPS data (Figure 2).

We can compare the calculated and experimental results. The DFT results above show that CO disproportionation on a nanosized iron cluster is a multistep process in which the rate-limiting step is the formation of $\mathrm{CO}_{2}$ from $\mathrm{O}$ and $\mathrm{CO}$. We studied different reaction channels, and the lowest energy channel showed a barrier of $1.04 \mathrm{eV}$, which, considering that the particle morphology and reaction conditions are different, is comparable to the value obtained from the kinetic investigations, with a barrier of $0.33 \mathrm{eV}$ for the overall process. The computed nanoparticle is very small and regular, and thus it has only a few types of active sites. In this sense it is probably rather unreactive. The larger clusters used in the experiments will have more active sites and some of them can have lower activation barriers. Even thought the difference between the experimental and simulated cluster is rather large, we wanted to emphasise that the calculations have given new information on the reactivity of nanoclusters, and comparisons to real-world systems are more relevant at lower temperatures.

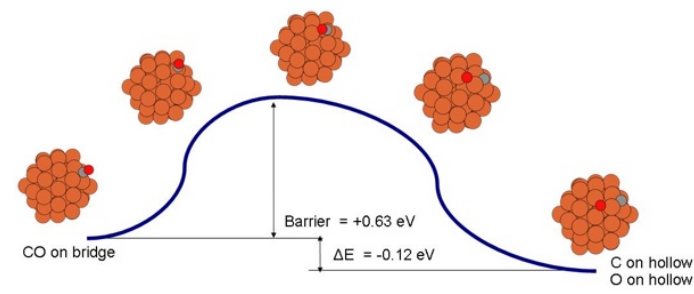

Figure 3 Calculated minimum energy paths for $\mathrm{CO}$ dissociation on a step of the $\mathrm{Fe}_{55}$ nanoparticle ( $\mathrm{Fe}$, brown; $\mathrm{C}$, grey; $\mathrm{O}$, red).

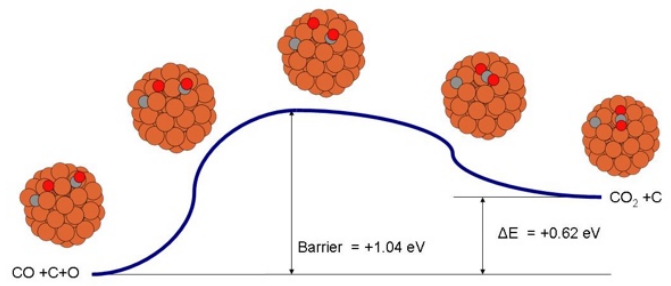

Figure 4 Calculated minimum energy paths for $\mathrm{CO}_{2}$ formation of the $\mathrm{Fe}_{55}$ nanoparticle (Fe, brown; $\mathrm{C}$, grey; $\mathrm{O}$, red). 
As stated above, the decomposition of metal carbides is regarded as the crucial step in the mechanism of CNT synthesis. To examine the role of this step in the overall process, the adsorption geometry of atomic carbon has been studied. The most stable geometries on and below the surface together with the calculated energetic path connecting them are depicted in Figures 5 and 6 . The energetic for the mobility of the carbon on the catalyser surface presents minor changes, both in terms of the barrier $(\leq 0.17 \mathrm{eV})$ and the energetic balance $(\approx 0.04 \mathrm{eV})$. This behaviour changes when possible reaction paths for the inclusion of atomic carbon under the surface of the $\mathrm{Fe}_{55}$ nanoparticle are considered (Figure 6). The obtained formation energy for a carbide-like structure is $-0.50 \mathrm{eV}$ with a barrierless process that presents an elementary rearrangement of atoms and molecules on the surface. Further inclusion of the atomic carbon in the cluster $(\Delta \mathrm{E}=+0.43$ $\mathrm{eV}$ ) requires overcoming a barrier of $0.51 \mathrm{eV}$.

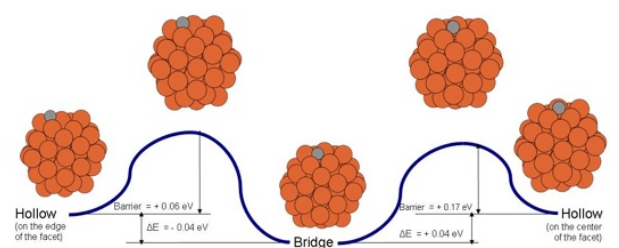

Figure 5 Calculated reaction path for the mobility of atomic $\mathrm{C}$ on the $\mathrm{Fe}_{55}$ nanoparticle.

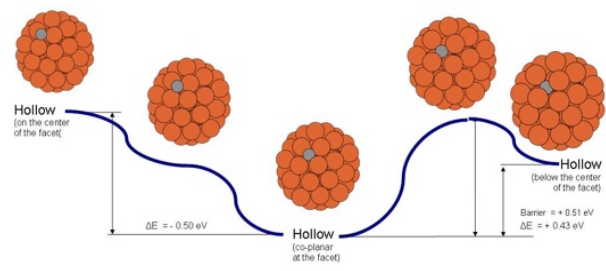

Figure 6 Calculated reaction path for the inclusion of atomic carbon under the surface of the $\mathrm{Fe}_{55}$ nanoparticle.

4 Conclusions We have presented an ab initio study of the $\mathrm{CO}$ disproportionation and $\mathrm{C}$ mobility on an iron nanoparticle. Overall, we see that the GPAW code is reliable for representing the high catalytic activity of small iron clusters. We have shown that the overall reaction barrier for CO disproportionation is relatively low and a suitable path for surface bound elemental carbon has been found. The barrier obtained from the kinetic study $\left(\mathrm{E}_{\mathrm{a}}=0.33 \mathrm{eV}\right)$ differs significantly from the value obtained computationally $\left(E_{a}=1.04 \mathrm{eV}\right)$. Apparently, the reason for this disagreement is related to the difference between the modelled particles and the actual morphology of the catalytic material during the experimental synthetic process. The real clusters have a similar geometry, but are bigger in size and most probably present highly reactive defects that cannot be modelled on a small nanocluster. Based on these findings, the study of larger clusters is envisaged to follow the present work.
Acknowledgements The authors would like to express their sincere thanks to Prof. J.K. Nørskov for useful discussions. The authors are grateful to M. Kupfer and M. Grobosch for the XPS facilities at IFW-Dresden. The project has mainly used the existing computational facilities available at CSC (Center for Scientific Computing), Espoo. This work has been supported in part by the European Commission under FP6 (STREP project BNC Tubes, contract number NMP4-CT-2006-03350), the Academy of Finland (project number 128445), and the Finnish TEKES GROCO Project (1298/31/08).

\section{References}

[1] R. Saito, G. Dresselhaus, and M.S. Dresselhaus, Physical properties of carbon nanotubes (Imperial College Press, London, 1998).

[2] M. Endo, Chemtech. 18, 568 (1988).

[3] T.W. Ebbesen, and P.M .Ajayan, Nature 358, 220 (1992).

[4] T. Guo, P. Nikolaev, A.G. Rinzer, D. Tomanek, D.T. Colberg, and R.E. Smalley, J. Phys. Chem. 99, 10694 (1995).

[5] P. Nikolaev, M.J. Bronikowski, R.K. Bradley, F. Rohmund, D.T. Colbert, K. Smith, and R.E. Smalley, Chem. Phys. Lett. 313, 91 (1999).

[6] A. Harutyunyan, B. Pradhan, U. Kim, G. Chen, and P. Eklund, Nano Lett. 2, 525 (2002).

[7] K. Hata, D.N. Futaba, K. Minuzo, T. Namai, M. Yumura, and S. Ijima, Science 306, 1362 (2004).

[8] C. Singh, M.S.P. Shaer, K.K.K. Koziol, I.A. Kinloch, and A.H. Windle, Chem. Phys. Lett. 372, 860 (2003).

[9] R.H. Baughman, A.A. Zakhidov, and W.A. de Heer, Science 297, 787 (2002).

[10] A.G. Nasibulin, A. Moisala, D.P. Brown, and E.I. Kauppinen, Carbon 41(14), 2711 (2003).

[11] N.R. Franklin, Y. Li, R.J. Chen, A. Javey, H. Dai, Appl. Phys. Lett. 79, 4571 (2001).

[12] A.G. Nasibulin, A. Moisala, D.P. Brown, H. Jiang, and E.I. Kauppinen, Chem. Phys. Lett. 402, 227 (2005).

[13] G. Lanzani, A.G. Nasibulin, K. Laasonen and E.I. Kauppinen, J. Phys. Chem. C 113, 12939-12942 (2010).

[14] G. Kresse, J. Hafner, Phys.: Condens. Matter 6, 8245 (1994).

[15] J.P. Perdew, J.A. Chevary, S.H. Vosko, K.A. Jackson, M.R. Pederson, D.J. Singh, C. Fiolhais, Phys. Rev. B 48, 4978 (1993).

[16] G. Kresse, J. Joubert, J. Phys. ReV. B 59, 1758 (1999).

[17] J. J. Mortensen, L. B. Hansen, and K. W. Jacobsen, Review B 71, 035109 (2005).

[18] B. Hammer, L. B. Hansen and J. K. Nørskov, Phys. Rev. B 59, 7413 (1999).

[19] G. Henkelman, B.P. Uberuaga, and H. Jonsson, J. Chem. Phys. 113, 9901 (2000).

[20] D.E. Jiang, and E.A. Carter, Surf. Sci. 570, 167 (2004).

[21] D.C. Sorescu, D.L. Thompson, M.M. Hurley, and C.F. Chabalowski, Phys. Rev. B 66, 035416 (2002).

[22] D. Borthwick, V. Fiorin, S.J. Jenkins, and D.A. King, Surf. Sci. 602 (13), 2325 (2008).

[23] Z.P. Liu, and P. Hu, J. Am. Chem. Soc. 123, 12596 (2001).

[24] P. Li, D.E. Miser, D. Rabiei, R.T. Yadav, and M.R. Hajaligo, App. Catal. B: Env. 43, 151 (2003).

[25] S. Colonna, S. De Rossi, M. Faticanti, I. Pettiti, and P. Porta, J. Mol. Catal. A: Chem. 187, 269 (2002). 